\title{
EXPERIMENTAL VERIFICATION OF KEY CROSS SECTIONS FOR PROMPT-GAMMA IMAGING IN PROTON THERAPY*
}

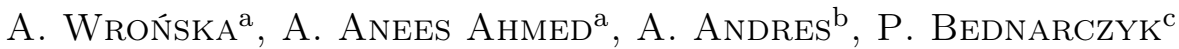 \\ J. Besuglow ${ }^{\mathrm{b}}$, G. Gazdowicz ${ }^{\mathrm{a}}$, K. Herweg ${ }^{\mathrm{b}}$, R. Hetzel ${ }^{\mathrm{b}}$

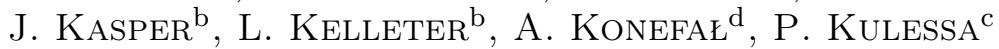

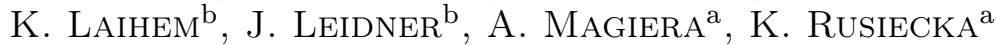 \\ D. Stachura ${ }^{\mathrm{a}}, \mathrm{A} \cdot \mathrm{STAhL}^{\mathrm{b}}, \mathrm{M} \cdot \mathrm{ZięBLiŃSKI}^{\mathrm{c}}$

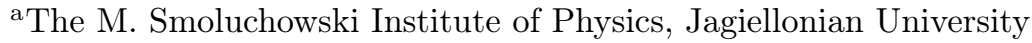 \\ Kraków, Poland \\ ${ }^{\mathrm{b}}$ RWTH Aachen University, Aachen, Germany \\ ${ }^{c}$ The H. Niewodniczański Institute of Nuclear Physics, PAN, Kraków, Poland \\ ${ }^{\mathrm{d}}$ Department of Nuclear Physics and Its Applications, Institute of Physics \\ University of Silesia, Katowice, Poland
}

\section{(Received August 21, 2017)}

We present experimental investigation of cross sections for processes crucial in view of prompt-gamma imaging. The prompt-gamma rays were produced from an interaction of a proton beam with different phantom materials composed of carbon, oxygen and hydrogen. The used target setup allowed precise selection of the investigated depth in the phantom. We studied details of the dependence of prompt-gamma yields on beam energy, detection angle and elemental composition of irradiated phantom. The analysis was focused on the discrete transitions with the largest cross sections: $4.44 \mathrm{MeV}$ in ${ }^{12} \mathrm{C}$ and $6.13 \mathrm{MeV}$ in ${ }^{16} \mathrm{O}$. The results are presented in form of profiles of the prompt-gamma yield as a function of depth. They are compared to calculations including different cross-section models. Obtained results are in agreement with the model exploiting cross-section data collected from the literature, but the comparison with the TALYS model shows discrepancies. In the latest experiment, special attention was paid to the shape of the distal fall-off. The width of that fall-off is directly linked to the resolution of prompt-gamma based methods of range verification. Preliminary results on the beam-energy dependence of this quantity are presented.

DOI:10.5506/APhysPolB.48.1631

* Presented at the $2^{\text {nd }}$ Jagiellonian Symposium on Fundamental and Applied Subatomic Physics, Kraków, Poland, June 3-11, 2017. 


\section{Introduction}

According to the findings of Eurostat and WHO, cancer is the second most common cause of death and morbidity in the European Union. The trend is unlikely to reverse in the near future. Therefore, beside development of new treatment methods, it is important to improve the methods that are currently in use. One of such methods is radiotherapy. The conventional X-ray radiotherapy was a subject of tremendous progress in recent years. Multi-field treatment plans allowed to reduce the dose delivered to the healthy tissue surrounding tumours. Despite that, in many cases, hadron therapy allows to achieve even better results. This is due to the unique character of proton beam interaction with the traversed material allowing better dose conformality as well as to larger biological effectiveness of hadron beams compared to X-rays. However, to fully exploit these features, one needs to develop methods for in vivo range verification, since hadron therapy is much more prone to errors caused by uncertainties of patient positioning, anatomical changes or translation of medical imaging into the maps of stopping power [1]. A family of proposed methods for range verification, such as prompt-gamma imaging, prompt-gamma spectroscopy, prompt-gamma timing, rely on the detection of prompt-gamma radiation emitted from a patient's body (for a recent review on the prompt-gamma based range verification methods, see e.g. $[2,3])$. The radiation is produced when tissue nuclei are excited by impinging beam ions and subsequently deexcite. Those gamma quanta carry undisturbed information from their place of origin, i.e. interaction region. Their yield depends on the beam energy, and thus it is spatially correlated with depth in the patient with respect to the Bragg peak position (BPP). In view of the elemental composition of the human tissue, the most important processes are: ${ }^{12} \mathrm{C}\left(p, p \gamma_{4.44 \mathrm{MeV}}\right)^{12} \mathrm{C}$, ${ }^{16} \mathrm{O}\left(p, X \gamma_{4.44 \mathrm{MeV}}\right){ }^{12} \mathrm{C}$ and ${ }^{16} \mathrm{O}\left(p, p \gamma_{6.13 \mathrm{MeV}}\right){ }^{16} \mathrm{O}$. The signals of those reactions in the prompt-gamma spectra are two peaks at $4.44 \mathrm{MeV}$ and $6.13 \mathrm{MeV}$. The aim of the project presented here was to study the cross sections for those processes and resulting gamma yields as functions of depth in phantoms in two experiments simulating the course of proton therapy. The results allow to verify models used in the simulation engines and offer insight into the intrinsic limitations of resolution of the prompt-gamma based range verification methods.

\section{Experiments}

After the pilot runs [4], two experiments were performed to study the prompt-gamma emission. The first experiment was performed at the Heidelberg Ion-Beam Therapy Center (HIT) using a proton beam accelerated to 70.54 or $130.87 \mathrm{MeV}$ kinetic energy. In this experiment, we studied the influence of the phantom elemental composition and detection angle on the 
shape of prompt-gamma depth profiles. The prompt-gamma depth profiles were determined for three phantom materials and two detection angles. The experiment was described in detail in [5]. The second experiment was performed recently at the Cyclotron Centre Bronowice (CCB) in Kraków and focused on studying the effects related to the change of beam energy. For this reason, the data were collected for the beam energies of 130, 180 and $230 \mathrm{MeV}$. At HIT, the beam was developed using a synchrotron, while CCB operates a cyclotron. This leads to slight differences in beam characteristics, e.g. its time structure. Also the beam exit from the ion pipe was different: at HIT, it was equipped with an active beam nozzle, at $\mathrm{CCB}$, it was a $50 \mu \mathrm{m}$ titanium foil. The conditions in the performed measurement series are summarized in Table I.

TABLE I

Overview of the conditions in the performed measurement series. Acronyms PMMA and POM stand for polymethyl methacrylate and polyoxymethylene, respectively. The atomic ratio carbon-to-oxygen for those materials is 5:2 (PMMA) and 1:1 (POM).

\begin{tabular}{cccl}
\hline \hline $\begin{array}{c}\text { Experimental } \\
\text { site }\end{array}$ & $\begin{array}{c}\text { Beam energy } \\
{[\mathrm{MeV}]}\end{array}$ & $\begin{array}{c}\text { Detection } \\
\text { angle }\end{array}$ & \multicolumn{1}{c}{$\begin{array}{c}\text { Phantom } \\
\text { materials }\end{array}$} \\
\hline \multirow{3}{*}{ HIT } & 70.54 & $90^{\circ}$ & PMMA, graphite, POM \\
& 70.54 & $120^{\circ}$ & PMMA, graphite \\
& 130.87 & $90^{\circ}$ & PMMA \\
& 130.87 & $120^{\circ}$ & PMMA, graphite \\
\hline \multirow{2}{*}{ CCB } & 130 & $90^{\circ}$ & PMMA \\
& 180 & $90^{\circ}$ & PMMA, graphite \\
& 230 & $90^{\circ}$ & PMMA, graphite
\end{tabular}

The experimental setup in the two beam times was similar and is schematically depicted in Fig. 1. In both cases, a high-purity germanium detector equipped with an active Compton shield was used to detect gamma radiation. In the CCB experiment, the radiation was detected also with a $\mathrm{LaBr}_{3}$ scintillation detector placed opposite to the germanium detector. The data from that detector, however, are still under analysis and are not presented here. The depth from which the radiation was reaching the detector was well-defined using a multi-part phantom, in which the investigated range of depth (thin slice) is spatially separated from the rest of the phantom. The depth of interest is varied by changing the position of one of the wedges, forming the thick part of the phantom. At HIT, this movement was in the horizontal plane, while at CCB, the target setup was modified and the movement was vertical. This solution offered more space which could be used to better shield the detector from the thick part of the target. As the depth 
range offered by the pair of wedges was insufficient to stop proton beams of higher energies $(\geq 180 \mathrm{MeV})$, for such measurements, the wedges were supplemented with a block extension inserted upstream of the wedges. All parts of the phantom were made of the same material.

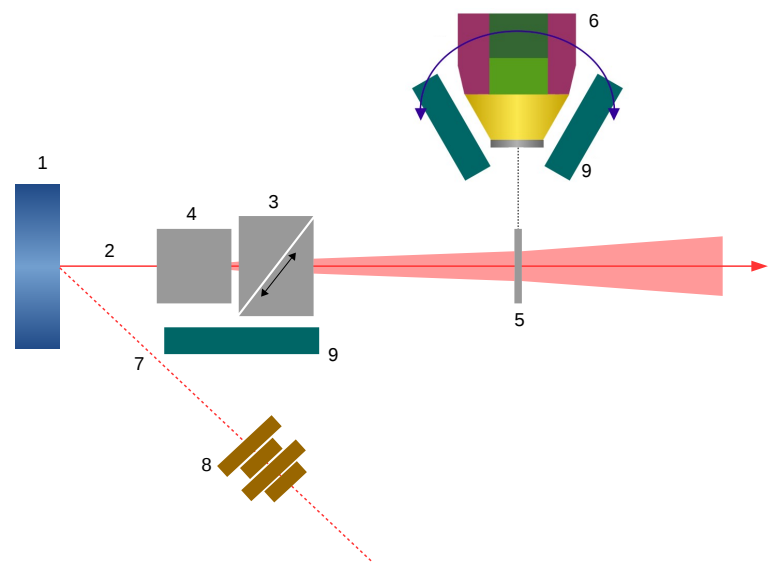

Fig. 1. A schematic top-view of the experimental setup: (1) beam pipe nozzle/exit window, (2) beam trajectory, (3) thick part of the phantom (wedges, arrow indicates direction of motion), (4) target extension necessary for larger beam energies, (5) thin phantom slice, (6) germanium detector, (7) trajectory of protons scattered on the exit nozzle material, (8) beam current monitors, (9) lead shield.

In order to see how the prompt-gamma yield is correlated with the depth in the phantom, for each set of conditions listed in Table I, a measurement series was taken, i.e. spectra were recorded for a number of values of target thickness around the proton range. A twin series without the thin slice in place was taken each time for the purpose of background study. The analysis showed that the background level at CCB was larger than at HIT, probably due to not optimal settings of the beam guiding system before the experimental hall. At similar conditions, beam energy of $130 \mathrm{MeV}$ and PMMA phantom, the signal-to-background ratio at maximum gamma emission were 3.1 at HIT and 1.8 at CCB.

Due to the small lateral dimensions of the thin slice $\left(5 \times 5 \mathrm{~cm}^{2}\right)$, not all protons leaving the ion pipe reached the slice. The fraction depends on the thickness of material upstream of the slice and the distances between the phantom parts. This effect was studied in Monte Carlo simulations and taken into account when comparing the obtained data to model calculations. The wedge-slice distance was different in both beam times, which led to shape differences of the profiles obtained under the same conditions at both centres. 


\section{Data analysis and results}

In the analysis, the spectra forming a measurement series were integrated in the regions of interest, i.e. region of $4.44 \mathrm{MeV}$ peak from carbon and $6.13 \mathrm{MeV}$ peak from oxygen. The integrals, after normalization by slice thickness, detector solid angle, number of impinging protons and correction for detection efficiency, plotted against target thickness formed depth profiles for each spectral line. Subsequently a twin profile for background series is produced and fitted with a smooth function. This smoothed background profile is subtracted from the signal profile, and the outcome represents the net effect from the slice material. The profiles determined from the HIT data are presented in Fig. 2, where the horizontal axis represents target thickness divided by proton range in the given material. Experimental data points are plotted together with results of model calculations using TALYS [6] cross sections as input. Although the distal fall-off in most cases is repro-

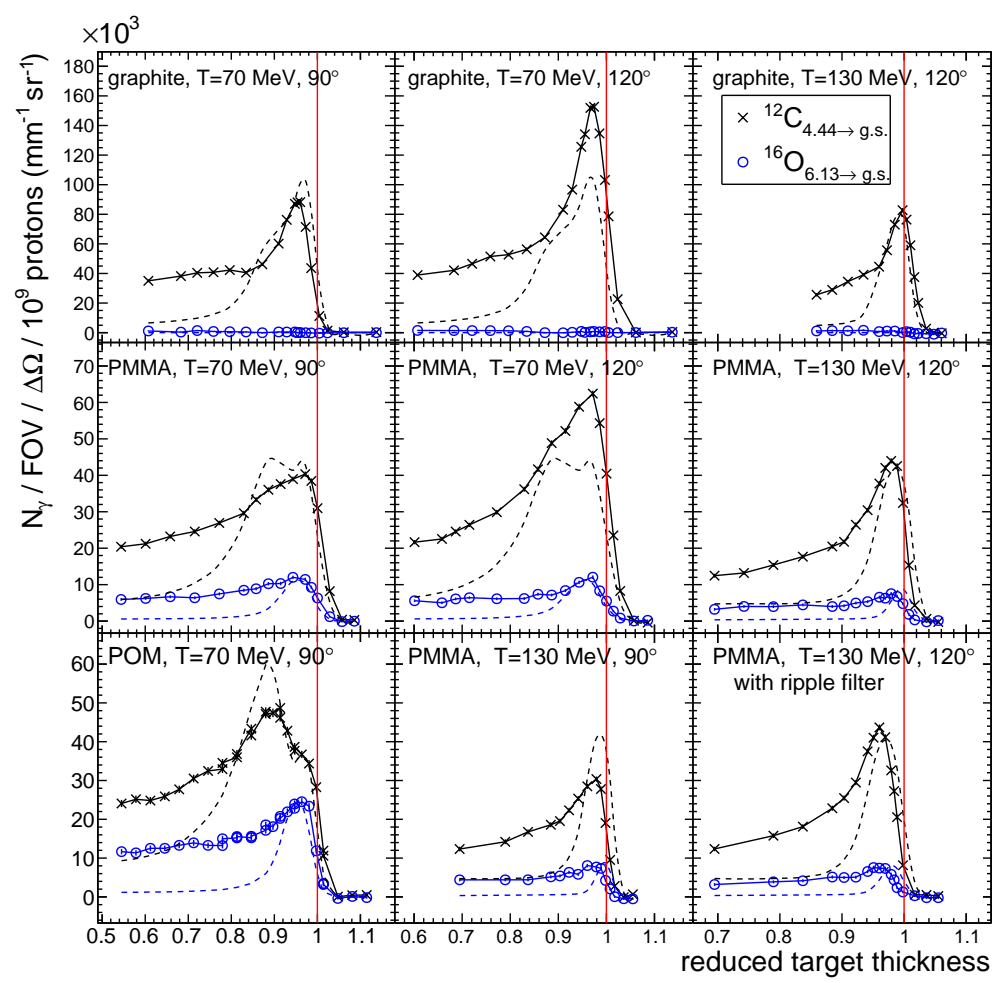

Fig. 2. (Colour on-line) Prompt-gamma depth profiles determined in the HIT experiment compared with predictions based on the TALYS results. The black crosses represent experimental data for $4.44 \mathrm{MeV}$ gammas and the black dashed lines show results of corresponding calculations based on cross sections from TALYS. The blue circles and lines refer to the results on $6.13 \mathrm{MeV}$ photons (reproduced from [5]). 
duced reasonably (horizontal shift results from systematic uncertainty of data points and is common for all points within one series), large discrepancies are found farther away from the BPP, i.e. for larger proton beam energies. Shape analysis of the profiles suggests that the contribution from the ${ }^{16} \mathrm{O}\left(p, X \gamma_{4.44 \mathrm{MeV}}\right){ }^{12} \mathrm{C}$ is overestimated in TALYS, the model also does not account for anisotropy in angular distribution of gamma emission. Similar comparison using available literature data for relevant cross sections as input shows better agreement (for details, see [5] and references therein). However, literature data are not available in full range covered by the experiment. It can be observed in Fig. 2 that the shapes of the investigated profiles depend on the phantom elemental composition.

The width of the distal fall-off is related to the smearing of the beam range resulting from energy straggling of the beam as well as from the width of the initial energy distribution of the beam. At the same time, this width determines the resolution limit which can be obtained with an ideal promptgamma imaging system. Therefore, special attention was paid to study this quantity as a function of beam energy. For this purpose, the data collected at various energies but otherwise similar conditions (PMMA phantom, $90^{\circ}$ detection angle) were analysed. The corresponding profiles for the $4.44 \mathrm{MeV}$ line are depicted in Fig. 3 (a). The fall-off width is defined as the difference of depths on which gamma yield drops from $90 \%$ to $10 \%$ of its maximal value (see Fig. 3 (b)). Its beam energy dependence is shown in Fig. 3 (c). It can be seen that while at lowest energies, the gamma yield drops steeply (on the path of about $1 \mathrm{~mm}$ ) when protons approach the BPP, for highest energies applied in proton therapy, this happens on an about six times longer path. All structures are smeared out compared to lower energies and the maximum in the prompt-gamma profile, well visible at $70 \mathrm{MeV}$ beam energy, is completely washed out.
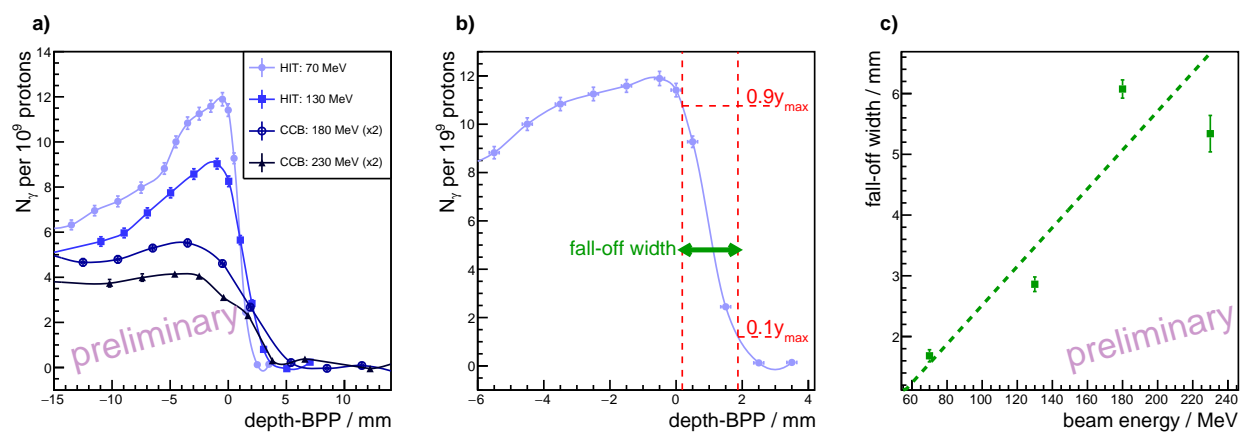

Fig. 3. From left to right: (a) depth profiles for the $4.44 \mathrm{MeV}$ line at various beam energies (see the legend); (b) definition of the fall-off width; (c) dependence of the fall-off width from the beam energy. 


\section{Summary}

Yields of prompt-gamma emission from ${ }^{12} \mathrm{C}_{4.44 \rightarrow \text { gs }}$ and ${ }^{16} \mathrm{O}_{6.13 \rightarrow \text { gs }}$ were measured in a series of experiments with proton beams of various energies impinging on phantoms differing by elemental composition. Obtained results are in line with existing cross-section data, but cannot be well-described using TALYS cross sections. The shape analysis of the depth profiles for the two investigated gamma transitions enables conclusions about the carbonto-oxygen ratio of the irradiated material. The distal fall-off visible in the depth profiles has a width which increases with beam energy. The main cause of this effect is beam energy straggling. Smearing of the distal falloff leads to deterioration of resolution of prompt-gamma range verification methods at higher beam energies.

The project was supported within the POMOST programme of the Foundation for Polish Science, co-financed from the European Union under the European Regional Development Fund as well as from the European Union's Horizon 2020 research and innovation programme under grant agreement No. 654002 (ENSAR2).

\section{REFERENCES}

[1] NuPECC report 2014: Nuclear Physics for Medicine, http: //www . nupecc.org/pub/npmed2014.pdf

[2] A.-C. Knopf, A. Lomax, Phys. Med. Biol. 58, R131 (2013).

[3] J. Krimmer, D. Dauvergne, J.M. Létang, É. Testa, Nucl. Instrum. Methods Phys. Res. A, in press, 2017, doi:10.1016/j.nima.2017.07.063.

[4] A. Wrońska et al., Acta Phys. Pol. B 46, 753 (2015).

[5] L. Kelleter et al., Phys. Medica 34, 7 (2017).

[6] NRG Petten 2016 TALYS 1.8, http://www.talys.eu/ 